\title{
Resistive RAM SET and RESET Switching Voltage Evaluation as an Entropy Source for Random Number Generation
}

\author{
Hussein Bazzi ${ }^{1 \& 2}$, Jeremy Postel-Pellerin ${ }^{1}$, Hassen Aziza ${ }^{1}$, Mathieu Moreau ${ }^{1}$, Adnan Harb ${ }^{3}$, Senior member, IEEE \\ ${ }^{1}$ Aix-Marseille Univ, Univ Toulon, CNRS, IM2NP, Marseille, France, ${ }^{2}$ Lebanese International University, Department of \\ Electrical and Electronics Engineering, Beirut, Lebanon, ${ }^{3}$ The International University of Beirut, Department of Electrical and \\ Electronics Engineering, Beirut, Lebanon
}

\begin{abstract}
The intrinsic variability of the switching parameters in resistive memories has been a major wall that limits their adoption as the next generation memories. In contrast, this natural stochasticity can be beneficial for other applications such as Random Number Generators (RNGs). This paper presents two RNG approaches based on a $130 \mathrm{~nm} \mathrm{HfO}_{2}$-based Resistive RAM (RRAM) memory array. The memory array is programmed with a voltage close to the median value of the SET (resp. RESET) voltage distribution to benefit from the SET (resp. RESET) voltage variability. In both cases, only a subset of the memory array is programmed, resulting in a stochastic distribution of cell resistance values. Resistance values are next converted into a bit stream and confronted to National Institute of Standards and Technology (NIST) test benchmarks.
\end{abstract}

Keywords- Resistive memories, Resistive RAM (RRAM), Random Number Generator (RNG), Stochastic switching, Memory array.

\section{INTRODUCTION}

A True Random Number Generator (TRNG) is a device that generates random numbers from a physical process, rather than by means of an algorithm. These processes are, in theory, stochastic and completely unpredictable. Conventional TRNG have been demonstrated based on Random Telegraph Noise (RTN) that occurs in semiconductors and ultra-thin gate oxide films $[1,2]$. In the last decade, TRNG-based resistive-switching devices have been widely studied by several research groups $[3,4]$. RRAMs have been utilized in several TRNG applications due to their appealing characteristics, including intrinsic stochastic nature [5], immunity to radiation [6], high endurance, CMOS compatibility [7] and low power consumption [8]. It is worth noticing that in literature, the generic term RRAM is often used to generically refer to both Oxide Random Access Memory (OxRAM) and Conductive-Bridging Random Access Memory (CBRAM) [9]. In the rest of the paper, OxRAM technology will be considered.

OxRAM devices suffer from a significant variability that can be divided into two categories, Device to Device (D2D) and Cycle to Cycle (C2C) variabilities [10]. This downside is analyzed and explored in this paper at a memory array level to assess the potential to use OxRAMs as TRNGs. Two sources of entropy are considered, the first one is based on the SET switching voltage variability and the second one is based on the RESET switching voltage variability. In both cases, programming voltages close to the SET and RESET voltage median distributions are applied. In these conditions, half of the cells in the array are expected to be in Low Resistive State (LRS) and the other half in High Resistive State (HRS). Thus, a stochastic distribution of resistance values across the array is anticipated. These resistance values are converted into logical values to generate a random bit stream with a perfect balance between 0 s and $1 \mathrm{~s}$ during the OxRAM cell READ operation by carefully trimming the reading voltages/currents. As the proposed TRNG combines $\mathrm{C} 2 \mathrm{C}$ and $\mathrm{D} 2 \mathrm{D}$ variabilities within a memory array, it overcomes limitations of state of the art OxRAM-based TRNGs which are mainly based on a single memory cell [11-13]. Moreover, using a memory array instead of a single cell turns the random number generation process more reliable.

In section II, experimental results obtained from a memory array based on an OxRAM technology are presented. In section III, the random number generation process is detailed and random numbers are generated after both SET and RESET operations and converted into a bit stream. In Section IV, the bit stream is confronted to the National Institute of Standards and Technology (NIST) test bench. Finally, section V provides some concluding remarks.

\section{OXRAM TECHNOLOGY}

\section{A. 1T1R memory cell}

Devices used in our TRNG circuit are bipolar OxRAMs where bipolar voltage sweeps are required to switch the resistive element. An OxRAM consists of two metallic electrodes that sandwich a thin dielectric layer serving as a storage medium. This structure can be easily integrated in the Back-End-Of-Line (BEOL) in combination with advanced CMOS technologies. As reported in [14], the MIM structure is integrated on top of Metal 4 copper layer $(\mathrm{Cu})$. A TiN Bottom Electrode (BE) is first deposited. Then, a $10 \mathrm{~nm}-\mathrm{HfO}_{2} / 10 \mathrm{~nm}-$ $\mathrm{Ti} / \mathrm{TiN}$ stack is added. In this configuration one MOS transistor is serially connected to an OxRAM cell. The select transistor acts as current limiting device. It controls the amount of current flowing through the cell according to its gate voltage value in order to prevent memory cell damage during programming operations [15]. Fig. 1 (a) and (b) present typical 1T1R OxRAM $\mathrm{I}-\mathrm{V}$ characteristics in linear scale. Based on these I-V curves, 
the memory cell behavior can be seen as follows: after an initial electro-FORMING step (not presented here), the memory element can be reversibly switched between a High Resistance State (HRS) and a Low Resistance State (LRS). Resistive switching in an OxRAM corresponds to an abrupt change between HRS and LRS. This resistance change is achieved by applying specific biases across the $1 \mathrm{~T} 1 \mathrm{R}$ cell (i.e. $\mathrm{V}_{\mathrm{SET}}$, to switch from HRS to LRS and $\mathrm{V}_{\text {RESET}}$, to switch from LRS to HRS). Fig. 1 (a) and (b) also highlight RESET and SET variabilities, here demonstrated after 7 consecutive RESET/SET operations applied to different cells (D2D in Fig. $1(\mathrm{a}))$ and to the same memory cell (C2C in Fig. 1(b)) across a memory array. Note that both $\mathrm{D} 2 \mathrm{D}$ and $\mathrm{C} 2 \mathrm{C}$ variabilities impact SET and RESET switching parameters. Table I presents the different voltage levels applied to the OxRAM cell during FORMING, RESET and SET operations. The whole experimental setup is based on a Keysight B1500 semiconductor parameter analyzer.

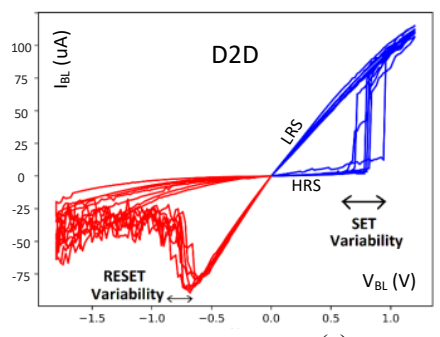

(a)

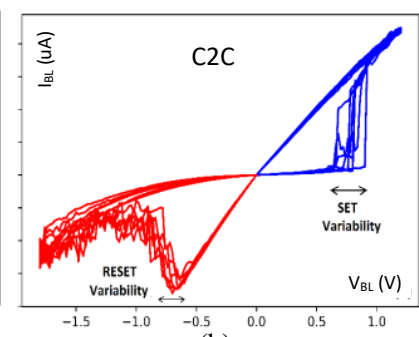

(b)
Fig. 1. I-V characteristics highlighting (a) D2D variability and (b) C2C variability. $V_{S E T}$ and $V_{\text {RESET }}$ variabilities are highlighted in both cases.

TABLE I. STANDARD OPERATING VOLTAGES (CELL LEVEL)

\begin{tabular}{|c|c|c|c|c|}
\hline & FORMING & RESET & SET & READ \\
\hline $\mathrm{WL}$ & $2 \mathrm{~V}$ & $4.5 \mathrm{~V}$ & $2 \mathrm{~V}$ & $4 \mathrm{~V}$ \\
\hline $\mathrm{BL}$ & $2 \mathrm{~V}$ & $0 \mathrm{~V}$ & $1.2 \mathrm{~V}$ & $0.2 \mathrm{~V}$ \\
\hline SL & $0 \mathrm{~V}$ & $1.8 \mathrm{~V}$ & $0 \mathrm{~V}$ & $0 \mathrm{~V}$ \\
\hline
\end{tabular}

The proposed random number generation methodology exploits the $\mathrm{HfO}_{2}$-based OxRAM variability (C2C and D2D) to generate a sequence of random bits. Indeed, $\mathrm{HfO}_{2}$-based OxRAM technology suffers from stochastic switching. These statistical fluctuations are generally attributed to the formation/dissolution of a Conductive Filament (CF) between metallic electrodes [16-18]. The number of defects and the distance between traps within the CF in both SET and RESET states are considered as the main causes of variability [19].

\section{B. 1T1R memory array}

Fig. 2 (a) presents the TRNG circuit which is basically a classical 1T1R array. Memory cells are grouped to form eight 8-bit memory words. Word Lines $\left(W L_{X}\right)$ are used to select the active row, Bit Lines $\left(B L_{X}\right)$ are used to select active columns during a SET operation and Source Lines $\left(S L_{X}\right)$ are used to RESET a whole memory word or an addressed cell. Fig. 2 (b) presents the layout view of the memory array. Due to the limited pin out of the probe card used in the experimental phase, only a $7 \times 7$ memory array is available for our experiments (subset of the $8 \times 8$ array). Based on this elementary memory array, the first step of the random number generation process is to extract SET and RESET switching voltage distributions over the array.

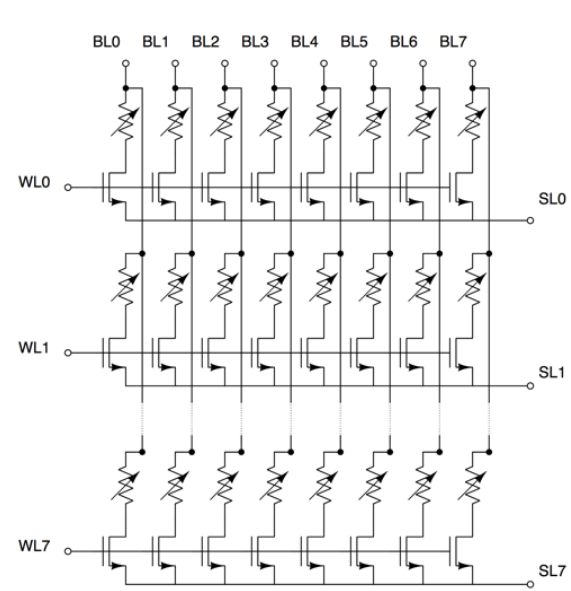

a)

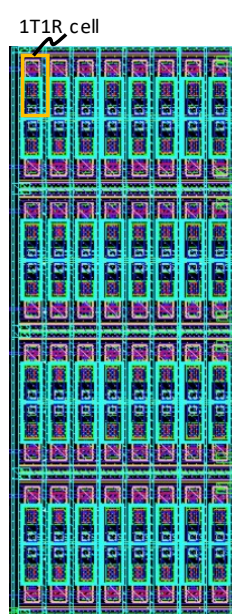

b)
Fig. 2. (a) 8x8 OxRAM memory array and (b) corresponding layout view.

To extract the RESET distribution, the memory array is first SET. Then, memory cells are RESET one by one to extract the RESET voltage value referred to as $V_{\text {RESET }}$. Regarding $V_{\text {SET }}$ distribution, the memory array is first RESET. Then, memory cells are SET one by one to extract the $\mathrm{V}_{\mathrm{SET}}$ threshold. The process is repeated 1000 times for both $\mathrm{V}_{\text {RESET }}$ and $\mathrm{V}_{\text {SET. }}$. Fig. 3 (resp. Fig. 4) presents the resulting $\mathrm{V}_{\text {RESET }}$ (resp. $\mathrm{V}_{\mathrm{SET}}$ ) distribution.

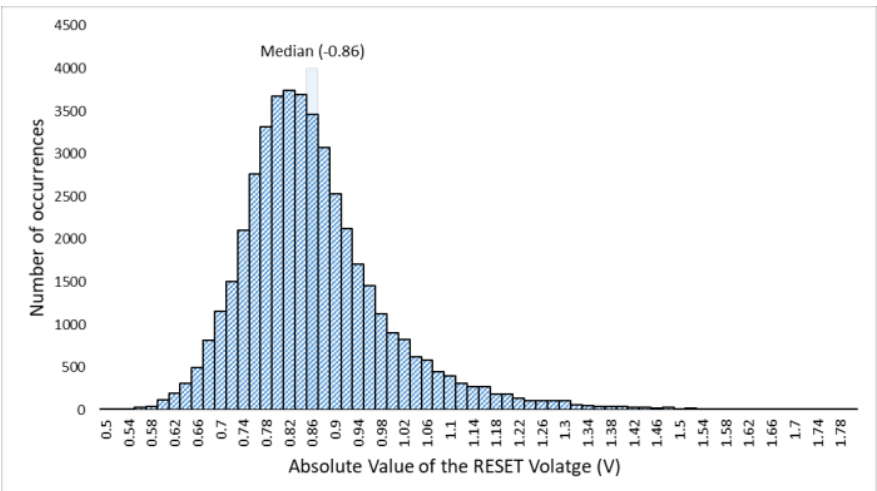

Fig. 3. RESET switching voltage $\left(\mathrm{V}_{\text {RESET }}\right)$ distribution obtained after 1000 SET/RESET cycles applied to the elementary memory array.

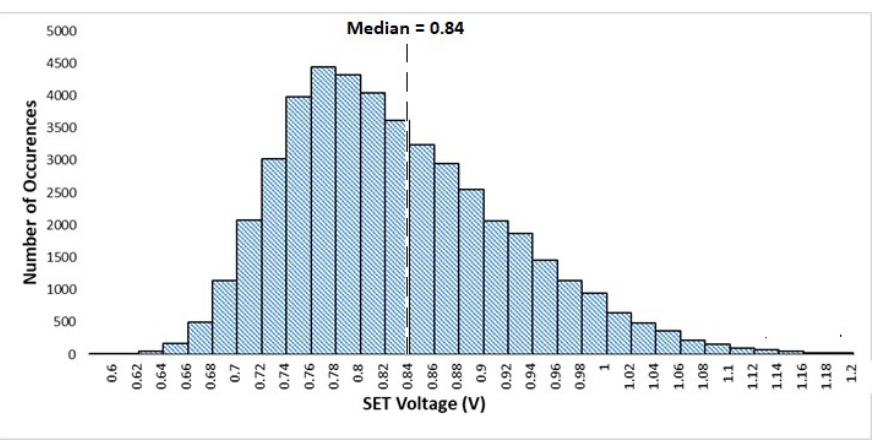

Fig. 4. SET switching voltage $\left(\mathrm{V}_{\mathrm{SET}}\right)$ distribution obtained after 1000 RESET/SET cycles applied to the elementary memory array. 
From these distributions, the median value of the RESET distribution referred to as $\mathrm{V}_{\text {RESET }} \mathrm{M}$ is extracted, as well as the median value of the SET distribution referred to as $\mathrm{V}_{\text {SET_M }}$. Results are summarized in Table II.

TABLE II. $V_{\text {RESET }} / \mathrm{V}_{\mathrm{SET}}$ DISTRIBUTION PARAMETERS

\begin{tabular}{cccc}
\hline \hline & Min & Median & Max \\
\hline RESET & $0.5 \mathrm{~V}$ & $\mathbf{- 0 . 8 6 ~ V}$ & $1.8 \mathrm{~V}$ \\
SET & $0.5 \mathrm{~V}$ & $\mathbf{0 . 8 4} \mathbf{V}$ & $1.2 \mathrm{~V}$ \\
\hline
\end{tabular}

\section{TRNG METHODOLOGY}

Note that $\mathrm{V}_{\text {RESET }} \mathrm{M}$ and $\mathrm{V}_{\text {SET }} \mathrm{M}$ extraction is a key point in the presented random number generation methodology as these values will be used as the conventional SET and RESET voltages for the next programming operations.

\section{A. RESET switching voltage-based $R N G$}

This RN generation process consists in setting the whole memory array and resetting each cell individually with $\mathrm{V}_{\text {RESET }} \mathrm{M}$ $=-0.86 \mathrm{~V}$ ("weak" RESET). As a result, some cells are switched to HRS, while others remain in LRS, resulting in a random distribution of resistance values. 100 runs are applied to the memory array to obtain a sufficiently large number of data (49x100). Fig. 5 presents an example of two 2D bitmaps obtained after two consecutive runs. Resistance values are represented by a greyscale from white (low values) to black (high values). Note that no spatial pattern reproduction has been detected after consecutive runs.
RUN N

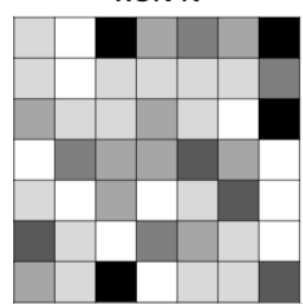

RUN N+1

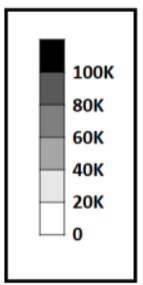

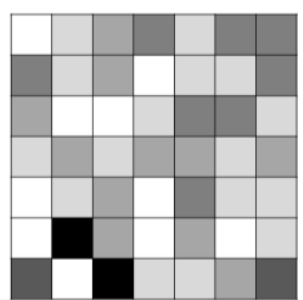

Fig. 5. Analog 2D bitmap (RESET) after Run N (left) and Run N+1 (right)

Fig. 6 presents the resistance distribution obtained after the weak RESET whose median value is equal to $32 \mathrm{k} \Omega$. This median is used in order to convert resistance values into a stream of bits presenting a perfect balance between ' 0 ' and ' 1 '. Despite external (temperature, etc.) or internal variations (degradation, etc.) the $50 \%$ ratio of ' 1 ' cells (and thus of ' 0 ' cells) has to be ensured. To this aim, real time voltage tracking techniques such as the one presented in [20] can be used. Indeed, NIST tests require an equal parity between ' 0 ' and ' 1 '. To convert resistance values into ' 0 ' and ' 1 ', all values lower than $R_{M}=32$ $\mathrm{k} \Omega$ (dashed line in Fig. 6) are associated with a ' 1 ' logical state while all higher values are associated with a ' 0 ' logical state.

The reading circuitry which basically consists in a simple biasing circuit with its output connected to a comparator is in charge of the distribution split [21]. Thus, the analog bitmaps presented in Fig. 5 are turned into logical bitmaps presented in Fig. 7. At run $\mathrm{N}$ we obtain 29 bits at ' 1 ' level (and thus 20 bits at ' 0 ' level) and at run $\mathrm{N}+1$ we obtain 23 bits at ' 1 ' level (and thus 26 bits at ' 0 ' level). Moreover, between the two presented runs 21 cells out of the 49 have changed their state and no systematic binary pattern has been observed between two consecutive runs.

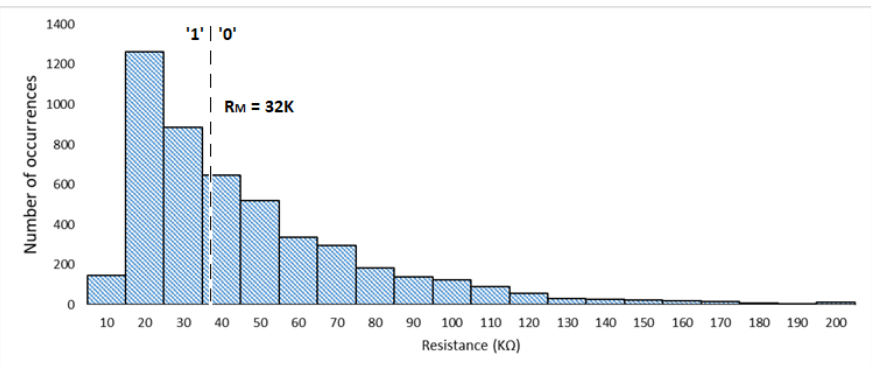

Fig. 6. Resistance distribution obtained after 100 consecutive memory array runs with a RESET value equals to $V_{\text {RESET_M }}(-0.86 \mathrm{~V})$.
RUN N

\begin{tabular}{|l|l|lllll|}
\hline 1 & 1 & 0 & 0 & 0 & 0 & 0 \\
\hline 1 & 1 & 1 & 0 & 1 & 0 & 0 \\
\hline 0 & 0 & 0 & 0 & 1 & 1 & 0 \\
\hline 1 & 0 & 0 & 0 & 0 & 0 & 1 \\
\hline 0 & 1 & 0 & 1 & 1 & 0 & 1 \\
0 & 1 & 1 & 0 & 0 & 0 & 1 \\
\hline 0 & 0 & 0 & 1 & 0 & 1 & 0 \\
\hline
\end{tabular}

RUN N+1

\begin{tabular}{|c|c|c|c|c|c|c|}
\hline 1 & 1 & 0 & 0 & 1 & 0 & 0 \\
\hline 0 & 1 & 0 & 1 & 1 & 0 & 0 \\
\hline 0 & 1 & 1 & 1 & 0 & 0 & 1 \\
\hline 1 & 0 & 0 & 0 & 0 & 1 & 0 \\
\hline 1 & 1 & 0 & 1 & 0 & 1 & 1 \\
\hline 1 & 0 & 0 & 1 & 0 & 1 & 1 \\
\hline 0 & 1 & 0 & 1 & 0 & 0 & 0 \\
\hline
\end{tabular}

Fig. 7. Digital 2D bitmap (RESET) after Run N (left) and Run N+1 (right)

\section{B. SET switching voltage-based $R N G$}

In this configuration, the $\mathrm{RN}$ generation process consists in resetting the whole memory array and setting each cell individually with $\mathrm{V}_{\mathrm{SET}_{-} \mathrm{M}}=0.84 \mathrm{~V}$ ("weak" SET). This study was reported in a previous work [22] where the SET voltage variability was exploited for RNG. Here also, some cells are switched to LRS state, while others remain in HRS state, resulting in a random distribution of resistance values. However, the resistance dispersion is larger. Fig. 8 presents an example of two 2D bitmaps obtained after two consecutive runs. Fig. 9 presents the resistance distribution whose median value is equal to $145 \mathrm{k} \Omega$ with values ranging from $10 \mathrm{k} \Omega$ to 1 $\mathrm{M} \Omega$. To convert resistance values into ' 0 ' and ' 1 ', all values lower than $\mathrm{R}_{\mathrm{M}}=145 \mathrm{k} \Omega$ (dashed line of Fig. 9) are associated with a ' 1 ' logical state while all higher values are associated with a ' 0 ' logical state. Logical bitmaps are presented in Fig. 10. One can notice that at run $\mathrm{N}$ we obtain 28 bits at ' 1 ' level (and thus 21 bits at ' 0 ' level) and at run $\mathrm{N}+1$ we obtain 28 bits at " 1 " level (and thus 21 bits at " 0 " level). Moreover, between the two presented consecutive runs, 13 cells out of the 49 have changed their state. Also, no systematic pattern is visible between the two runs. 

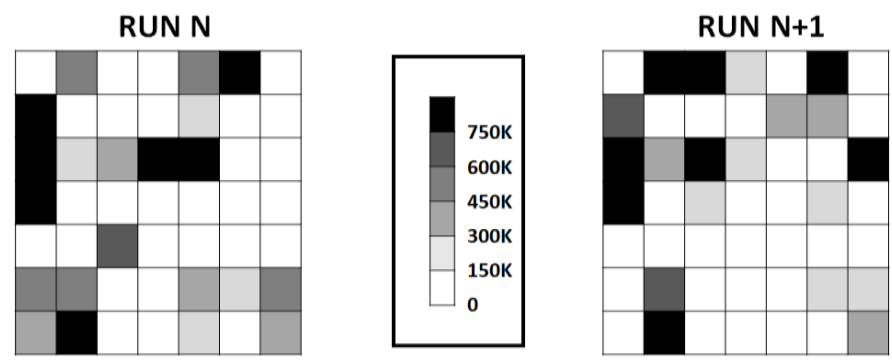

Fig. 8. Analog 2D bitmap (SET) after Run N (left) and Run N+1 (right)

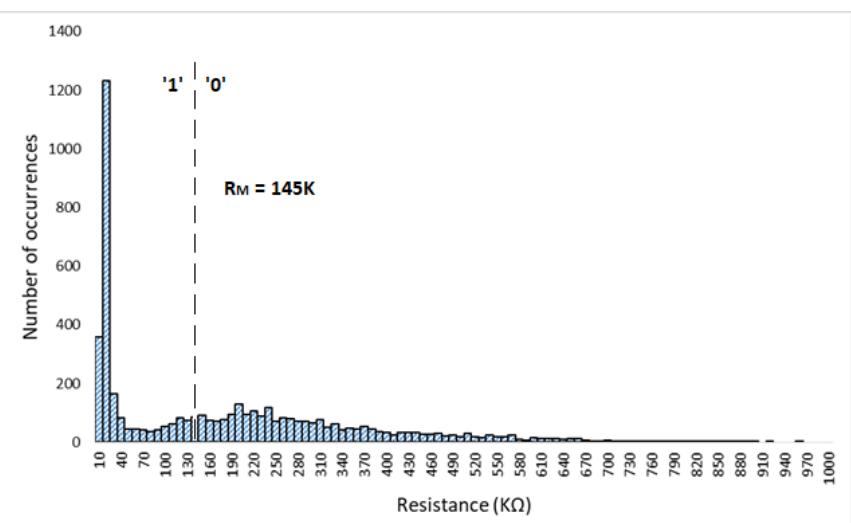

Fig. 9. Resistance distribution after 100 consecutive memory array runs (SET) [22]
RUN N

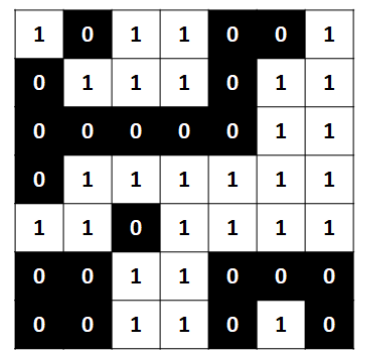

RUN N+1

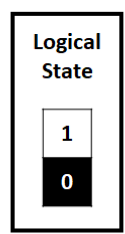

Fig. 10. Digital 2D bitmap (SET) after Run N (left) and Run N+1 (right)

\section{NIST TEST SUITE RESUlts}

The random numbers ( 4.9 kbits) generated after 100 runs and extracted from distributions presented in Fig. 6 (obtained after weak RESET) and Fig. 9 (obtained after weak SET) are verified against a statistical test suite. The statistical calculation is performed based on the NIST test suite for random and pseudorandom number generators for cryptographic applications [23]. For the distribution obtain after a weak RESET, the bit stream passes 9 NIST tests over 15 (see Table III). For the distribution obtained after a weak SET, the bit stream passes 11 NIST tests over 15 (see Table IV). It should be noted that these results are obtained without any post-processing step.

Tests 05-Binary Matrix Rank, 08-Overlapping Template Matching, 09-Maurer's Universal Statistical and 10-Linear Complexity are failed for both RESET and SET distributions. Indeed, these tests need a larger number of bits as recommended in [23]. Test 12-Approximate Entropy checks the frequency of all possible overlapping $\mathrm{m}$-bit patterns across the entire sequence. The purpose of the test is to compare the frequency of overlapping blocks of two consecutive/adjacent lengths ( $\mathrm{m}$ and $\mathrm{m}+1$ ) against the expected result for a random sequence, ensuring the quality of the random number sequence. Only the SET switching voltage-based bit stream passes this test ( $p$-value $>0.01$ ). Same goes for test 6-Discrete Fourier Transform or DFT used to detect periodic features (i.e. repetitive patterns that are near each other).

TABLE III. NIST TEST SUITE RESULtS AFTER WEAK RESET

\begin{tabular}{lcc}
\hline \multicolumn{1}{c}{ Test } & p-value & Result \\
\hline 01-Frequency & 0.8639 & Pass \\
\hline 02-Frequency within a block & 0.0872 & Pass \\
\hline 03-Runs & 0.7314 & Pass \\
\hline 04-Longest Run of ones in a block & 0.2693 & Pass \\
\hline 05-Binary Matrix Rank & - & Fail \\
\hline 06-Discrete Fourier Transform & - & Fail \\
\hline 07-Non-Overlapping Template & 0.5209 & Pass \\
\hline 08-Overlapping Template Matching & - & Fail \\
\hline 09-Maurer's Universal Statistical & - & Fail \\
\hline 10-Linear Complexity & - & Fail \\
\hline 11-Serial & 0.4781 & Pass \\
\hline 12-Approximate Entropy & - & Fail \\
\hline 13-Cumulative Sums & 0.1627 & Pass \\
\hline 14-Random Excursions & 0.3540 & Pass \\
\hline 15-Random Excursions Variant & 0.0726 & Pass \\
\hline \multicolumn{2}{c}{ Global NIST Score } & 9/15 \\
\hline \hline
\end{tabular}

TABLE IV. NIST TEST SUITE RESUlts AFTER WEAK SET

\begin{tabular}{lcc}
\hline \multicolumn{1}{c}{ Test } & p-value & Result \\
\hline 01-Frequency & 0.1736 & Pass \\
\hline 02-Frequency within a block & 0.6711 & Pass \\
\hline 03-Runs & 0.8157 & Pass \\
\hline 04-Longest Run of ones in a block & 0.9077 & Pass \\
\hline 05-Binary Matrix Rank & - & Fail \\
\hline 06-Discrete Fourier Transform & 0.8623 & Pass \\
\hline 07-Non-Overlapping Template & 0.9970 & Pass \\
\hline 08-Overlapping Template Matching & - & Fail \\
\hline 09-Maurer's Universal Statistical & - & Fail \\
\hline 10-Linear Complexity & - & Fail \\
\hline 11-Serial & 0.4921 & Pass \\
\hline 12-Approximate Entropy & 0.9999 & Pass \\
\hline 13-Cumulative Sums & 0.3248 & Pass \\
\hline 14-Random Excursions & 0.4072 & Pass \\
\hline 15-Random Excursions Variant & 0.5929 & Pass \\
\hline \multicolumn{2}{l}{ Global NIST Score } & $\mathbf{1 1 / 1 5}$ \\
\hline \hline
\end{tabular}

\section{A. Comparison and Discussion}

Table $\mathrm{V}$ shows a comparison between the two proposed methods. The comparison is based on five parameters including NIST test result, resistance median distribution, expected energy/cell, expected bit-rate and the use of a post-processing step. The resistance distribution median value for RESET (32 $\mathrm{k} \Omega)$ is lower than the SET one $(145 \mathrm{k} \Omega)$. This difference has a straight impact on the energy consumption during the read operation preceding the bit stream generation. Thus, assuming that the RNG is properly configured (weak SET and weak RESET operation achieved and threshold resistances properly extracted), the energy/cell during RNG use is evaluated to $0.125 \mathrm{fJ}$ and $0.027 \mathrm{fJ}$ after weak RESET and weak SET respectively (values computed for a $100 \mathrm{~ns}$ read pulse at $0.2 \mathrm{~V}$ considering the average post weak RESET and post weak SET 
resistances). Regarding the expected bit-rate, it has been demonstrated that read time in the $10 \mathrm{~ns}$ order can be achieved for hafnium oxide devices resulting in bit rate in the order of $100 \mathrm{Mb} / \mathrm{s}$ [24]. Finally, the proposed RNGs do not include postprocessing circuits such as XOR gates [25] and thus, can be improved in terms of randomness quality.

Table V. Comparison Between The Two Proposed Methods

\begin{tabular}{cccccc}
\hline \hline & $\begin{array}{c}\text { NIST } \\
\text { Results }\end{array}$ & $\begin{array}{c}\text { Median } \\
\text { Resistance }\end{array}$ & $\begin{array}{c}\text { Energy/cell } \\
(\mathrm{fJ})\end{array}$ & $\begin{array}{c}\text { Bit-rate } \\
(\mathrm{Mb} / \mathrm{s})\end{array}$ & $\begin{array}{c}\text { Post- } \\
\text { processing }\end{array}$ \\
\hline RESET & 9 & $32 \mathrm{k} \Omega$ & 0.125 & 100 & No \\
SET & 11 & $145 \mathrm{k} \Omega$ & 0.027 & 100 & No \\
\hline \hline
\end{tabular}

From a physical point of view, generally, the HRS is more affected by switching variability, because the applied negative voltage results in a partial depletion of defects from the CF. Any variation in the conduction path and in the energy barrier impacts exponentially the resistance, therefore the statistical variations of HRS resistance are relatively large. Moreover, the resistance cumulative distributions slightly increases with $\mathrm{V}_{\text {RESET, as }}$ previously demonstrated by experiments and simulations of HfOx-based RRAM [26]. On the other hand, resistance fluctuations in the LRS are generally due to geometrical variations in the $\mathrm{CF}$ cross section, which affect LRS resistance only linearly resulting in tighter resistance distributions. Regarding randomness, no correlation with variability is observed as the weak SET programming approach produce better randomness than the weak RESET programming approach.

\section{CONCLUSION}

The proposed TRNG is implemented based on an elementary array of 1T1R OxRAM cells. Based on two programming method exploiting weak RESET and weak SET resistance distributions, it is possible to obtain random numbers. However, it has been demonstrated that the capability to produce randomness is higher for weak SET resistance distributions compared to weak RESET resistance distributions. These results have been confirmed after NIST tests. Indeed, the obtained bit streams have passed 9 and 11 NIST tests over 15 without any post-processing step for RESET and SET respectively.

\section{ACKNOWLEDGMENT}

The authors wish to acknowledge the support from AixMarseille University, and Lebanese International University.

\section{REFERENCES}

[1] R. Brederlow, et al., "A low-power true random number generator using random telegraph noise of single oxide-traps," in ISSCC Tech. Dig., San Francisco, CA, USA, 2006, pp. 1666-1675.

[2] D. Lelmini, et al., "Resistance-Dependent Amplitude of Random Telegraph-Signal Noise in Resistive Switching Memories," Applied Physics Letters, vol. 96, no. 5, 2010, p. 053503.

[3] Y. Wang, et al., "A novel true random number generator design leveraging emerging memristor technology," in Proc. GLSVLSI, Pittsburgh, PA, USA, 2015, pp. 271-276.

[4] S. Sahay, et al., "Recent trend in hardware security exploiting hybrid CMOS-resistive memory circuits" in Semiconductor Science and Technology, vol. 32, no. 12, 2017, pp. 123001.
[5] V. Van der Leest, et al., "Hardware intrinsic security to protect value in the mobile market. ISSE 2014 Securing Electronic Business Processes (Springer Vieweg, Wiesbaden, 2014).

[6] Y. Wang, et al., "Highly Stable Radiation-Hardened Resistive-Switching Memory," in IEEE Electron Device Letters, vol. 31, no. 12, pp. 1470 1472, Dec. 2010.

[7] D. Ielmini, et al., "Physical modeling of voltage-driven resistive switching in oxide RRAM," 2012 IEEE International Integrated Reliability Workshop Final Report, South Lake Tahoe, CA, 2012, pp. 9-15.

[8] C. Cagli, et al., "Study of the Energy Consumption Optimization on RRAM Memory Array for SCM Applications," 2017 IEEE International Memory Workshop (IMW), Monterey, CA, 2017, pp. 1-4.

[9] H. Wang, et al., "Overview of Resistive Random Access Memory (RRAM): Materials, Filament Mechanisms, Performance Optimization, and Prospects," Rapid Research Letters, vol. 13, no. 9, 2019, p. 1900073.

[10] A. Fantini, et al., "Intrinsic switching variability in HfO2 RRAM," in Proc. IMW, Monterey, CA, 2013, pp. 30-33.

[11] S. Balatti, et al., "True random number generation by variability of resistive switching in oxide-based devices,” IEEE J. Emerg. Sel. Topics Circuits Syst., vol. 5, no. 2, pp. 214-221, June 2015.

[12] S. Sahay, et al., "OxRAM RNG Circuits Exploiting Multiple Undesirable Nanoscale Phenomena," in IEEE Transactions on Nanotechnology, vol. 16, no. 4, pp. 560-566, July 2017.

[13] H. Jiang, et al., "A novel true random number generator based on a stochastic diffusive memristor," Nature communications, vol. 8 , p. 882 , Oct. 2017.

[14] G. Molas, et al., "Resistive Memories (RRAM) Variability: Challenges and Solutions," ECS Transactions, vol. 86, no. 3, pp. 35-47, 2018.

[15] A. Grossi, et al., "Experimental Investigation of 4-kb RRAM Arrays Programming Conditions Suitable for TCAM," IEEE Trans. VLSI Syst., vol. 26, no. 12, pp. 2599-2607, Dec. 2018.

[16] S. Larentis, et al., "Resistive switching by voltage-driven ion migration in bipolar RRAM - Part II: Modeling", in IEEE Trans. Electron Devices, vol. 59, no. 9, pp. 2468-2475, Jun. 2012.

[17] E. Miranda, "Compact model for the major and minor hysteretic I-V loops in nonlinear memristive devices", in IEEE Transactions on Nanotechnology, vol. 14, no. 5, pp. 787-789, July 2015.

[18] L. Larcher, et al., "Microscopic understanding and modeling of $\mathrm{HfO}_{2}$ RRAM device physics", in Proc. IEDM, San Francisco, CA, USA, 2012, pp. 20.1.1-20.1.4.

[19] F. M. Puglisi, et al., "Bipolar Resistive RAM Based on HfO2: Physics, Compact Modeling, and Variability Control," IEEE J. Emerg. Sel. Topics Circuits and Syst., vol. 6, no. 2, pp. 171-184, June 2016.

[20] W. H. Choi et al., "A magnetic tunnel junction based true random number generator with conditional perturb and real-time output probability tracking," in Proc. IEEE Int. Electron Devices Meeting (IEDM), Dec. 2014, pp. 12.5.1-12.5.4.

[21] H. Aziza, et al., "A built-in self-test structure (BIST) for resistive RAMs characterization: Application to bipolar OxRRAM. Solid-State Electronics", 103, pp.73-78, 2015.

[22] J. Postel-Pellerin, et al., "True Random Number Generation Exploiting SET Voltage Variability in Resistive RAM Memory Arrays." 2019 19th Non-Volatile Memory Technology Symposium (NVMTS), In press.

[23] A. Rukhin, et al., "A Statistical Test Suite for Random and Pseudorandom Number Generators for Cryptographic Applications," Nat. Inst. Standards Technol., Gaithersburg, MD, USA, Pub. 800-22, 2001.

[24] Boubacar Traore. Investigation of HfO2-based resistive RAM cells by electrical characterization and atomistic simulations. Micro and nanotechnologies/Microelectronics. Université Grenoble Alpes, 2015.

[25] D. Kaya, et al.., "Random Number Generators Based on Irregular Sampling and Fibonacci-Galois Ring Oscillators." IEEE Transactions on Circuits and Systems II: Express Briefs, vol. 66, no. 10, 2019, pp. 17181722.

[26] S. Ambrogio, et al., "Statistical fluctuations in HfOx resistive- switching memory (RRAM): Part I - Set/Reset variability," IEEE Trans. Electron Devices, vol. 61, no. 8, pp. 2912-2919, Aug. 2014. 